\title{
Study of Proportional Guidance Trajectory Based on Guiding Principle
}

\author{
Wang Xin, Guo Xin and Yao Jun \\ Shenyang Ligong University, Shenyang, 110159, China \\ sylaizh@163.com
}

\begin{abstract}
In missile guidance systems, classical proportional navigation law has been used most widely. But with the increasing of the maneuverability of the targets, the classical proportional navigation guidance law has been unable to meet the requirements of people on the ballistic parameters. So this paper will further study the algorithm error of guidance law. Based on the previous theoretical results and the working principle of aircraft in the homing guidance system, this paper studies a guidance trajectory guidance principle which based on the ratio, and has carried on the analysis and the simulation theory. And simulation accuracy analysis has been carried on with practical examples. The results show that the method is put forward to effectively reduce the guided ballistic calculation method of error.
\end{abstract}

Keywords: Guidance Law, Guiding Principle, Proportional Guidance, Robust Geometric Guidance

\section{Introduction}

In the process of aircraft flying actually, due to various constraints, the guidance system every sampling step to obtain a play yard azimuth and distance. So, based on the numerical expression of proportional guidance equation trajectory, using creates a continuous equation, difference equation to describe the process of discrete, thereby increasing the method error of the algorithm itself. This paper introduces the guiding principle of aircraft and the proportional guidance equation is deduced, which leads to the calculating method based on the guidance of the principle of trajectory, thus directly using the discrete expressions to describe the process, to avoid the use of continuous equation difference formula of the method error. Finally simulation accuracy analysis has been carried on the with practical examples. The results show that the method is put forward to effectively reduced the guided ballistic calculation method of error [1-4].

\section{Guiding Principle of Aircraft}

Aircraft proportional guidance trajectory and target trajectory is shown in Figure 1. In the figure $D D_{1}, D D_{2} \ldots D_{3} D_{4}$ are aircraft trajectory; $M^{M_{1}}, M_{1} M_{2} \ldots M_{3} M_{4}$ are target trajectory. When the aircraft flew to control period of the end $D$, target is located in $M$. The direction of the line of sight is $D M$. The Aircraft initial trajectory Angle is $\theta=0^{\circ}$. The initial heading is $D D_{1}$. Through a sampling step length $T$, the aircraft arrives in $D_{1}$. The target arrive in $M_{1}$.At this time, the direction of the line of sight is $\overrightarrow{D_{1} M_{1}}$. Aircraft at point $D_{1}$ direction of the line of sight rotation angular velocity is $\dot{a}_{i}=\left(\overrightarrow{D_{1} M_{1}-\vec{D} \boldsymbol{M}}\right)^{\prime T}$. According to the 
proportional guidance equation $\dot{\theta}=\kappa \dot{q}$, calculate aircraft trajectory angle is $\theta_{1}$. And then determine the aircraft's new course is $\overrightarrow{D_{1} D_{2}}$.After a time step $T$, aircraft arrived at $D_{2}$ point and the direction of the line of the rotating angular velocity is

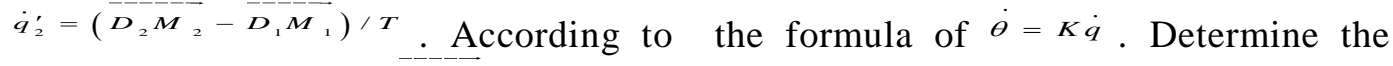
aircraft's new course is $\overrightarrow{D_{2} D_{3}}$. So constantly, until hitting their targets [5-8].

\section{Proportional Guidance Equation}

As shown in Figure 2, at certain moment target located is at point $M$, and aircraft is located at point $O$.The target velocity is ${ }^{V_{m}}$; the aircraft velocity is $V$. Both in the same plane. In the figure, $R$ is the relative distance between the aircraft and the target, and $q$ is the Angle of the line of sight, and $\eta_{\mathrm{m}}$ and $\eta$ is the aircraft heading angle. ${ }^{\theta}{ }_{m}$ and $\theta$ are the goal and the vehicle velocity vector and the reference line angle[9-10].

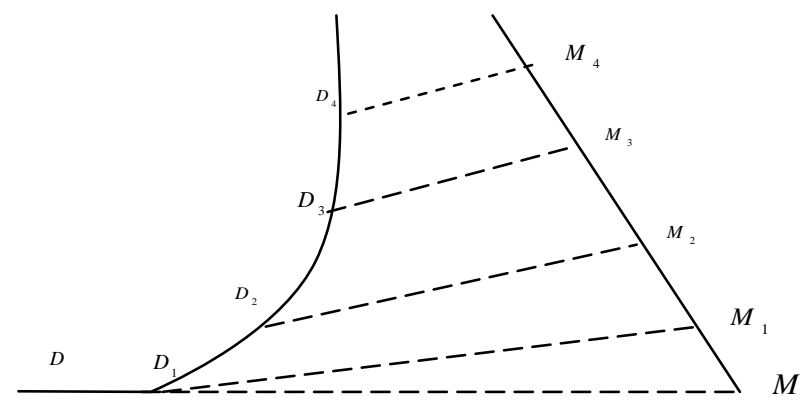

Figure 1. Proportional Guidance Trajectory Diagram

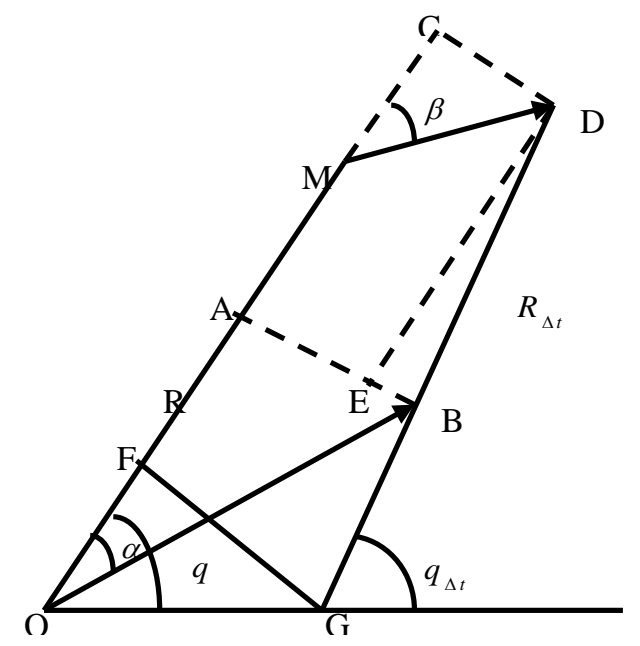

Figure 2. Single Step Movement within the Diagram

Derivation: As guides $A B \perp O M, C D \perp O M$, and meet in $\mathrm{OM}$ extension of C. $D E \perp A B$. Hereon, $|O M|=R,|O B|=a,|M D|=b$ 
Obvious,

$$
\begin{gathered}
|A M|=|O M|-|O A|=R-a \square_{\cos } \alpha \\
|A C|=|A M|+|M C|=R-a \square_{\cos } \alpha+b \square_{\cos \beta}
\end{gathered}
$$

Because of the quadrilateral ACDE as rectangles, and $|B E|=|A B|-|A E|=|A B|-|C D|$, thus

$$
|D B|=\sqrt{|D E|^{2}+|B E|^{2}}=\sqrt{R^{2}+b^{2}+a^{2}-2 a b \square(\alpha-\beta)+2 R(b \square \cos \beta-a \square \cos \alpha)}
$$

$$
R=\frac{d y}{d t}=\lim _{\Delta t \rightarrow 0} \frac{|D B|-|O M|}{\Delta t}=v_{m} \square_{\cos }\left(q-\theta_{m}\right)-v \square \cos (q-\theta)
$$

Obvious $\angle D B A=\angle D G F, \angle O G F=\frac{\pi}{2}-q$,

$$
\begin{aligned}
& q_{\Delta t}=\pi-\angle D G F-\angle O G F=\frac{\pi}{2}+q-\arctan \left(\frac{R-v \square_{\cos \alpha+v_{m}} \square_{\Delta t} \square_{\cos \beta} \beta}{{ }_{v} \square_{\Delta t} \square_{\sin } \alpha-v_{m} \square_{\Delta t} \square_{\sin } \beta}\right) \\
& \dot{q}=\lim _{\Delta t \rightarrow 0} \frac{q_{\Delta t}-q}{\Delta t}=\left(v \square \sin \alpha-v_{m} \square \sin \beta\right) / R
\end{aligned}
$$

By (2) and (4) simultaneous, proportional guidance equation is obtained

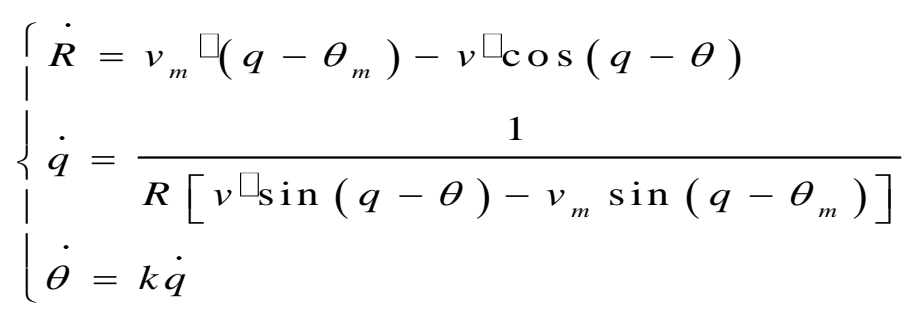

When $\mathrm{R}=0$ to hit the target.

\section{Guidance Ballistic Based On Guidance of Principle}

Based on the principle of proportional guidance, (1) and (3) are used to establish a homing trajectory,

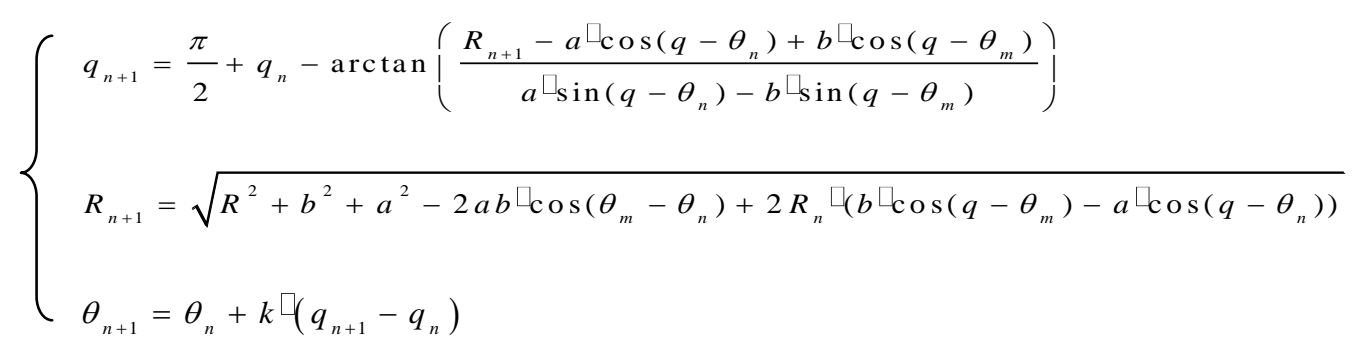


In this: ${ }^{\square}$ is sampling for aircraft time step; $a=v^{\square} \Delta t$ is for the aircraft in $\Delta t$ time displacement; ${ }^{b}=v_{m}{ }^{\square} t$ is the target displacement of time; ${ }^{\theta}$ is target motion direction angle; ${ }^{\theta_{n}}$ is aircraft trajectory angle; ${ }_{n}$ is target angle of sight; $k$ is proportional guidance coefficient[[11-15].

\section{Trajectory Simulation}

According to the deduced formula (4.6), the MATLAB software is used for simulation problem of two-dimensional proportional guidance. Assuming that aircraft control paragraph to end point $(0,0)$, the velocity is $300 \mathrm{~m} / \mathrm{s}$. The initial heading along the $\mathrm{x}$ axis towards the right, When fly the end point of section $\mathrm{x}$ line machine to control the coordinates of the target $(5000,10000)$; target nvelocity is $120 \mathrm{~m} / \mathrm{s}$; desired track is $60^{\circ}$. The simulation time step is $0.1 \mathrm{~s} . K=2$. The ballistic is shown in Figure 3 and 4.

Figure 3 and Figure 4 respectively proportional guidance coefficient of $K=2$ and $K=6$ vehicle and target motion trajectory diagram, more can be found that the size of the proportional guidance coefficient $K$, which has a great influence on the whole trajectory curvature radius. $K$ proportional guidance coefficient is smaller, guided ballistic the bending; $K$, the greater the guidance trajectory is straight.

The method of this article is comparing with classical proportional guidance trajectory. Take $K=2$, as shown in Figure 5, the classical proportional guidance trajectory in this paper. Methods of ballistic are shown in Figure 6.

Comparing Figure 5 and Figure 6, we can find that the two methods in the algorithm error when $K=2$, obviously, has led to a ballistic difference is very big. Classical proportional guidance law of ballistic curve curvature is bigger, the method of the ballistic curve is flat. Ballistic trajectory has been leveled off, in the front slightly bent, ballistic not big fluctuations, flat. Therefore, based on the guiding principle of proportional guidance trajectory better tracking stability, it is does not have the advantages of the classical proportional guidance law.

The following two methods to produce the algorithm error are analyzed. Respectively on the classical proportional guidance and the article adopts the method of the data processing, take different proportion coefficient $K$ is obtained relevant error such as shown in Table 1, Table 2 and Table 3, it is concluded that the approximation.

Table 1. $K=2$, Error of Method in this Paper And Classical Proportional Guidance

\begin{tabular}{cccccccc}
\hline $\mathrm{T}$ & 0 & 5 & 10 & 15 & 20 & 25 & 30 \\
error & 0 & 0.02 & 0.07 & 0.12 & 0.18 & 0.25 & 0.36 \\
\hline $\mathrm{T}$ & 35 & 40 & 45 & 50 & 54 & 57 & 60 \\
error & 0.36 & 0.48 & 0.63 & 0.81 & 1.13 & 1.18 & 1.2 \\
\hline
\end{tabular}

According to the data in Table 1, image processing using MATLAB software, the result is shown in Figure 7. 


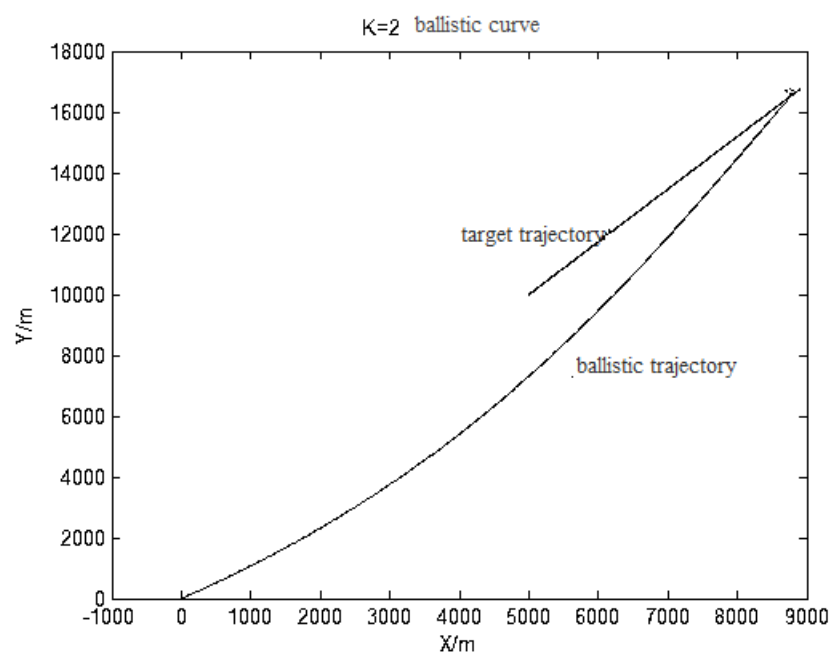

Figure 3. $\mathrm{K}=2$, Aircraft and the Target Motion Trajectory

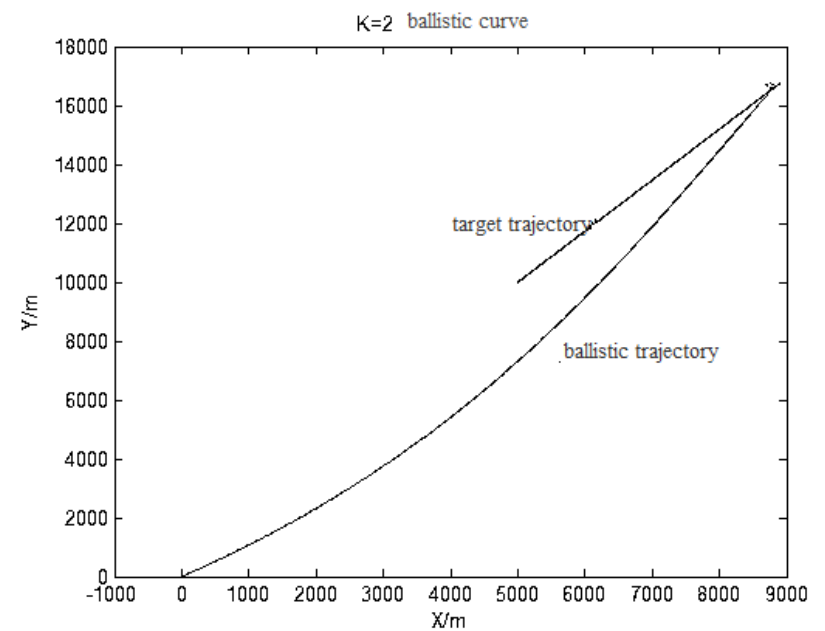

Figure 4. $\mathrm{K}=6$ Aircraft and the Target Motion Trajectory

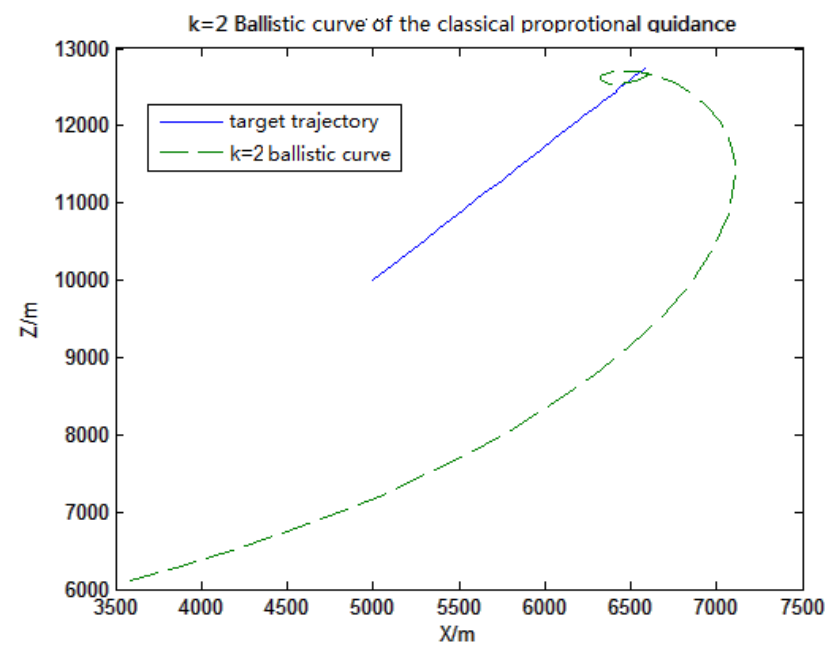

Figure 5. K=2 Classical Proportional Guidance Trajectory 


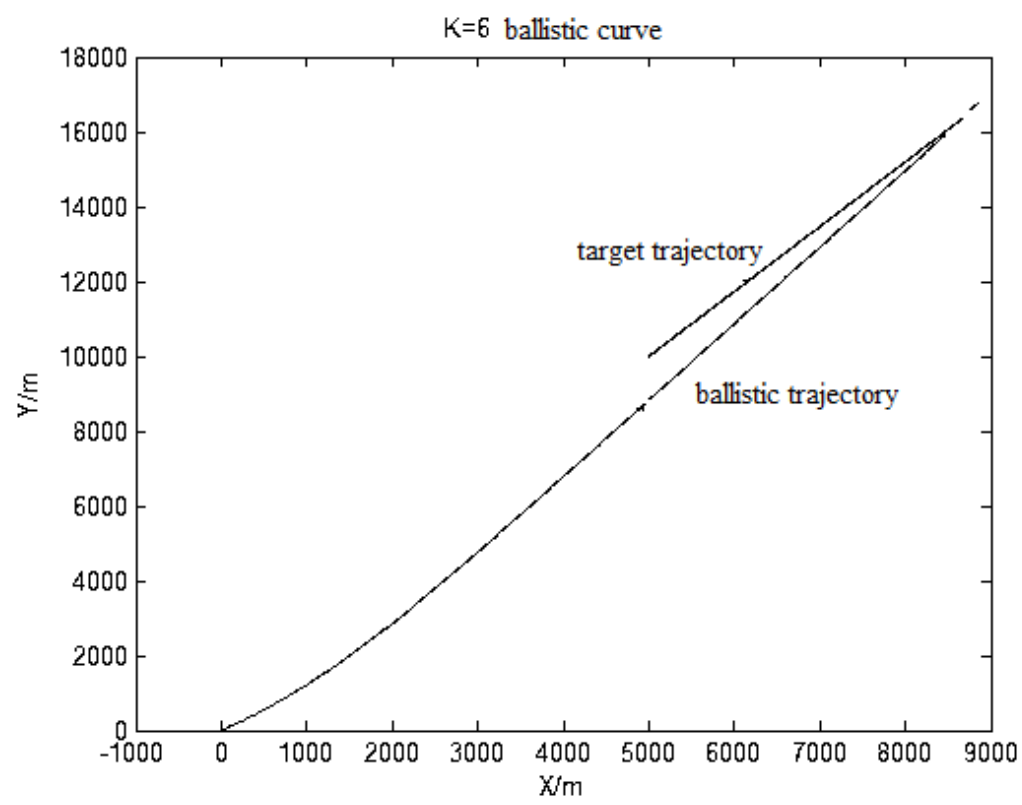

Figure 6. K=2 Ballistic Diagram Method in This Paper

Table 2. $K=4$, Error of Method in This Paper and Classical Proportional Guidance

\begin{tabular}{cccccccc}
\hline $\mathrm{T}$ & 0 & 5 & 10 & 15 & 20 & 25 & 30 \\
error & 0 & 0.03 & 0.09 & 0.14 & 0.21 & 0.27 & 0.32 \\
\hline $\mathrm{T}$ & 35 & 40 & 45 & 50 & 54 & 57 & 60 \\
error & 0.35 & 0.37 & 0.371 & 0.372 & 0.373 & 0.374 & 0.375 \\
\hline
\end{tabular}

To Table 1, Table 2 and Table 3 using the MATLAB software for processing, the result is shown in Figure 8.

Table 3. $K=6$, Error of Method in This Paper and Classical Proportional Guidance

\begin{tabular}{cccccccc}
\hline $\mathrm{T}$ & 0 & 5 & 10 & 15 & 20 & 25 & 30 \\
error & 0 & 0.03 & 0.09 & 0.14 & 0.18 & 0.19 & 0.2 \\
\hline $\mathrm{T}$ & 35 & 40 & 45 & 50 & 54 & 57 & 60 \\
error & 0.21 & 0.22 & 0.221 & 0.222 & 0.223 & 0.224 & 0.225 \\
\hline
\end{tabular}

From Figure 8 comparison can be found that with the increase of time ${ }^{t}$, the algorithm error is also increased in a certain scope, reaches a certain value, the algorithm error is stabilized, similar to a fixed value. With the increase of $K$ value, the method error is small. That is the size of the method error value and is inversely proportional to the size of the $K$ value. 


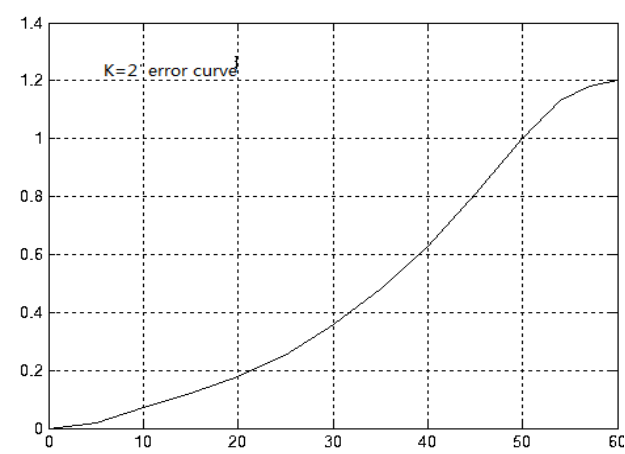

Figure 7. $\mathrm{K}=2$ Error Curve

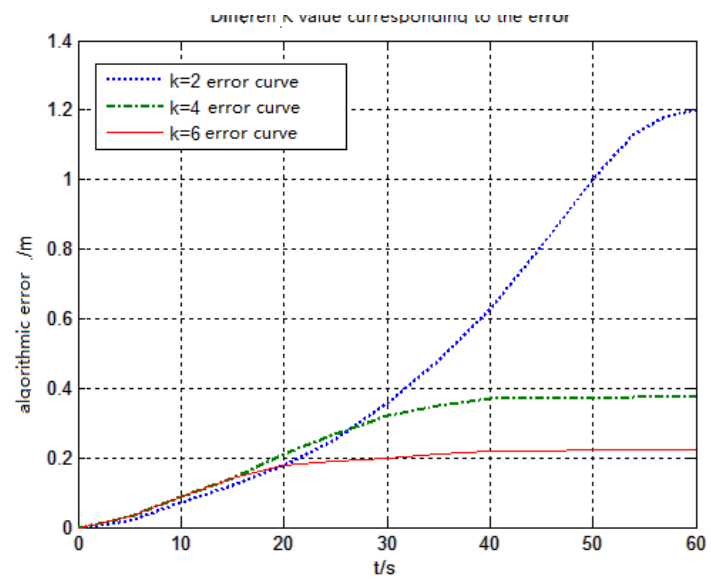

Figure 8. Error and Direct the Diagram of Coefficient $\mathrm{K}$

Below again respectively on classical proportional guidance and the article adopts the method of the data processing, take different faster than ${ }^{c}$ obtained the related error such as shown in Table 4.4, Table 4.5 and Table 4.4, it is concluded that the approximation. Through these data to the next step algorithm error analysis, thus we can draw the error and speed ratio ${ }^{c}$.

Table 4. $C=1.67$, Error of Method in this Paper and Classical Proportional Guidance

\begin{tabular}{ccccccccc}
\hline $\mathrm{t}$ & 0 & 5 & 10 & 15 & 20 & 25 & 30 & 35 \\
error & 0 & 0.006 & 0.03 & 0.07 & 0.11 & 0.15 & 0.18 & 0.19 \\
\hline 40 & 45 & 50 & 55 & 60 & 65 & 70 & 75 & 80 \\
0.191 & 0.192 & 0.193 & 0.194 & 0.195 & 0.196 & 0.197 & 0.198 & 0.199 \\
\hline
\end{tabular}

Table 5. $\mathrm{C}=2.5$, Error of Method in this Paper and Classical Proportional Guidance

\begin{tabular}{ccccccc}
\hline $\mathrm{t}$ & 0 & 5 & 10 & 15 & 20 & 25 \\
error & 0 & 0.02 & 0.05 & 0.1 & 0.18 & 0.26 \\
\hline 30 & 35 & 40 & 45 & 50 & 55 & 60 \\
0.32 & 0.34 & 0.347 & 0.348 & 0.349 & 0.35 & 0.351 \\
\hline
\end{tabular}


Table 6. $\mathrm{C}=5$, Error of Method in this Paper and Classical Proportional Guidance

\begin{tabular}{cccccc}
\hline $\mathrm{T}$ & 0 & 5 & 10 & 15 & 20 \\
Error & 0 & 0.04 & 0.1 & 0.18 & 0.28 \\
\hline 25 & 30 & 32 & 35 & 40 & 45 \\
0.43 & 0.55 & 0.577 & 0.588 & 0.595 & 0.60 \\
\hline
\end{tabular}

Will different ${ }^{c}$ and MATLAB software for processing, the relationship between the error form the results is shown in Figure 9.

As can be seen from the Figure 7, the error is proportional to the speed ratio ${ }^{c}$. With the increase of time ${ }^{t}$, the algorithm error is also increased in a certain scope, after reaching a certain value tends to be stable, approximate to a fixed value. Compare Figure 7 and Figure 6, found in Figure 6 the algorithm error value is greater than the algorithm error in Figure 7, is the size of the proportion coefficient $K$, the error of the method have a larger impact. And speed ratio ${ }^{c}$ changes on the guidance method error of ballistic has obvious influence.

\section{Conclusions}

This paper is for proportional guidance principle based on aircraft. Strictly proportional guidance equation is deduced, and raises the based on the guidance of the principle of recursive formula for calculating trajectory, and finally ballistic simulation is carried out according to the two-dimensional proportional guidance problem, and further analyzes the proportional guidance coefficient and speed ratio on the influence of method error. Through the contrast we can see that the size of the proportional guidance coefficient $K$, on the whole trajectory curvature radius and the influence of method error is bigger. Speed ratio c change on guided ballistic method has obvious influence, and $\mathrm{c}$ in time will be different. Therefore, in the vehicle guidance system design, considering various factors, optimization of related parameters, further enhance the combat effectiveness of aircraft.

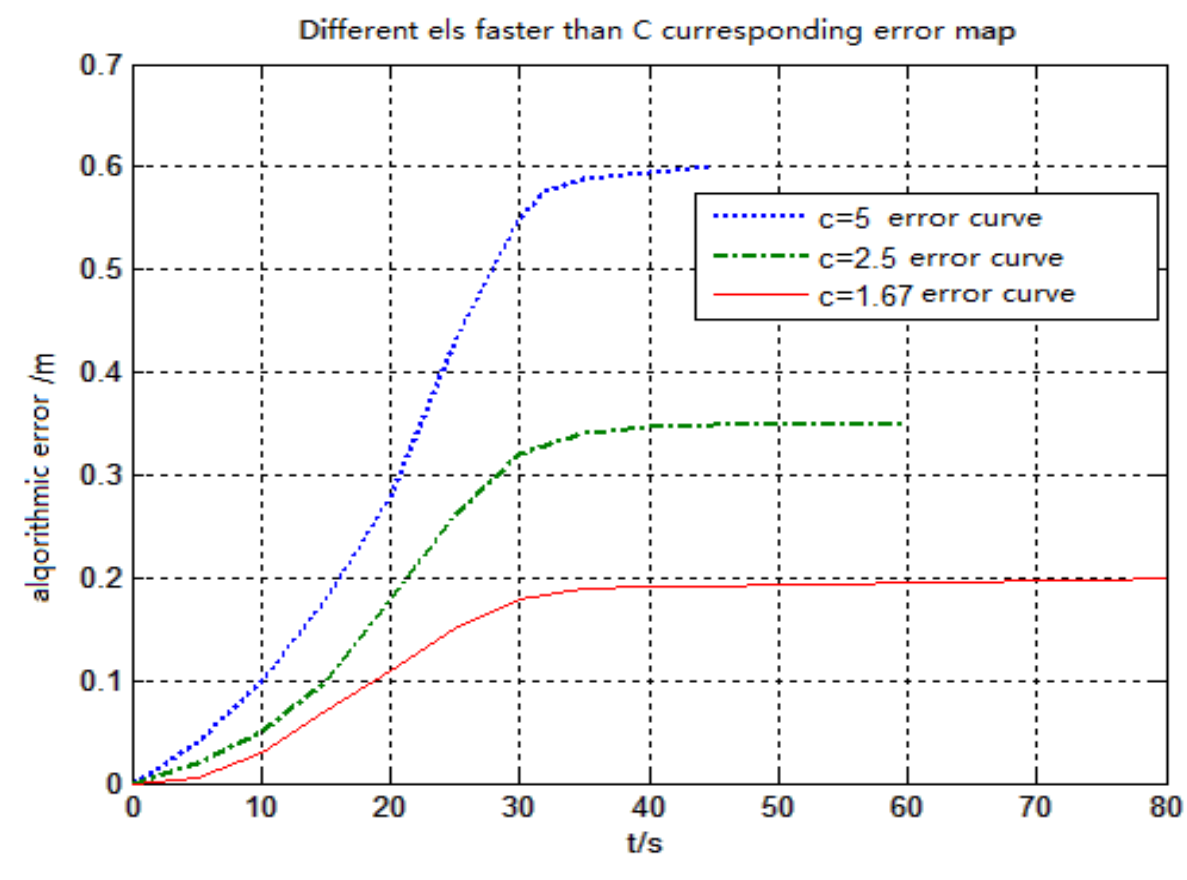

Figure 9. Relationship of Error and Speed Ratio C 


\section{References}

[1] Yuan P J. Solution of True Proportional Navigation with Maneuvering and Nonmaneuvering Targets. IEEE Translations on AES,1995,31(1): 469-474.

[2] GaoShang. "Simulation on missile ideal trajectory under on proportion guide method", Computer Engineering and Design, 2003, 24(4).

[3] Zhang, Y., Ma and P.B.: Three-dimensional guidance law with impact angle and impact time constraints. Acta Aeronaut. Astronaut. Sin. 29(4), 1020-1026 (2008)

[4] Song, J.M., Zhang and T.Q.: The passive homing missile's variable structure guidance law with terminal impact angular costraints. J. Ballist. 13(1), 16-21 (2001)

[5] Cao, S.B., Jiang, C.S., Guang, S.Y. and Chen, M.: Research on the vertical intercept terminal guidance system of TV-command-guidance air-to-ground missile. J. Astronaut.25 (4), 393-397 (2004)

[6] Yin, Y.X., Yang, M., Wu and P.: Three dimensional guidance laws with attack angle constraint for maneuverable target. Solid Rocket. Technol. 33(3), 237-241 (2010)

[7] Zhou Jiyuan, Tong Youtang and ZhangLei. Simulation and Analysis of Three-dimensional Trajectory on Typical Guide Method[J].Ship Electronic Engineering,2008(2):110-112.

[8] Gao Shang. "Proportional Navigation ideal trajectory simulation", Computer Engineering and Design, 2003, 24(8):66-67.

[9] Wang Ya-fei, Fang Yang-wang and Zhou Xiao-bin. "Proportional Navigation Research and Development", Fire Control and Command Control, 2007,32(10) : 8-11.

[10] Mehrandezh M. "Robotic interception of moving objectsusing an augmented ideal proportional navigation guid-ance technique", IEEE Transactions on Systems,Man, and Cybernetics,2000,30(3):231244.

[11] Sim Y C,Leng S B and Subramaniam V. "An all-aspect near-optimal guidance law", Dynamics and Control,2000,10(2):165-177.

[12] Duc Cuong Quach, Shuang Huang, Quan Yin and Chunjie Zhou. An improved Direct Adaptive Fuzzy controller for an uncertain DC Motor Speed Control System[J]. TELKOMNIKA Indonesian Journal of Electrical Engineering. Vol 11, No 2,February 2013.

[13] Zhang Zhongnan, Tong Youtang and Zhang Weifeng, Research on the Proportional Guidance Trajectory Simulation[J].Tactical Missile Technology,2005(2):56-59.

[14] Shoucri, R.M. "Elliptical Orbit with Variable Angular Momentum", Journal of Guidance, Control, and Dynamics, 1995,18(5):1213-1215.

[15] Zhang Jian-wei, Huang Shu-cai and Han Zhao-chao. "Proportional Guidance Trajectory Based on MATLAB simulation analysis", Tactical Missile Technology, 2009, 16(3) : 60-64.

\section{Authors}

Wang Xin(1973-), female, Doctor, Vice professor, and field is missile guidance.

Guo Xin(1990-), male, Master graduate student.

YAO Jun(1962-), male, Doctor, Professor, and field is missile guidance. 
International Journal of Hybrid Information Technology Vol.8, No.9 (2015) 